- Von praktisch großer Bedeutung ist darüber hinaus, dass sich die Träger von UNESCO-Global Geoparks aktiv in die räumliche Planung einbringen, um die Berücksichtigung ihrer Belange gegenüber konkurrierenden Raumnutzungsansprüchen, wie etwa der Windenergie, zu forcieren, weil die Windenergienutzung grundsätzlich durch die räumliche Planung vorbereitet wird und die räumliche Planungsebene daher eine frühzeitige Möglichkeit bietet, die eigenen Belange in Entscheidungsprozesse einzubringen. Träger von UNESCO-Global Geoparks sollten geltend machen, als Träger öffentlicher Belange bei Planungsprozessen beteiligt zu werden. Soweit Träger von UNESCOGlobal Geoparks privatrechtlich organisiert sind, wäre $\mathrm{zu}$ erwägen, eine Anerkennung als Umweltvereinigung gem. $\$ 3$ Umweltrechtsbehelfsgesetz bzw. gemäß des jeweiligen Landesrechts zu beantragen, um ggf. Klagerechte im Hinblick auf einen wirksamen primären Schutz des Geoparks geltend machen zu können.
Open Access. Dieser Artikel wird unter der Creative Commons Namensnennung 4.0 International Lizenz veröffentlicht, welche die Nutzung, Vervielfältigung, Bearbeitung, Verbreitung und Wiedergabe in jeglichem Medium und Format erlaubt, sofern Sie den/die ursprünglichen Autor(en) und die Quelle ordnungsgemäß nennen, einen Link zur Creative Commons Lizenz beifügen und angeben, ob Änderungen vorgenommen wurden.

Die in diesem Artikel enthaltenen Bilder und sonstiges Drittmaterial unterliegen ebenfalls der genannten Creative Commons Lizenz, sofern sich aus der Abbildungslegende nichts anderes ergibt. Sofern das betreffende Material nicht unter der genannten Creative Commons Lizenz steht und die betreffende Handlung nicht nach gesetzlichen Vorschriften erlaubt ist, ist für die oben aufgeführten Weiterverwendungen des Materials die Einwilligung des jeweiligen Rechteinhabers einzuholen.

Weitere Details zur Lizenz entnehmen Sie bitte der Lizenzinformation auf http://creativecommons.org/licenses/by/4.0/deed.de.

Open access funding provided by Projekt DEAL.

\title{
https://doi.org/10.1007/s10357-020-3694-1
}

\section{Flächenverbrauch im Recht: 30-Hektar-Ziel und Flächenzertifikatehandel}

\author{
Hendrik Hamacher
}

C Der/die Autor(en) 2020

Die Problematik des Flächenverbrauches ist seit Jahrzehnten in Deutschland bekannt. Zugleich wird diese in Politik und Gesellschaft nur geringfügig berücksichtigt. Dabei ist der Schutz und der nachhaltige Umgang der Ressource Fläche aus vielfältigen Gründen von immenser Bedeutung. Gleichwohl führen die gegenwärtigen Methoden und Instrumente nicht dazu, die politisch verabschiedeten Ziele zu erreichen. Im Rahmen dieses Beitrages werden auf Grundlage der aktuellen Gegebenheiten, neue Möglichkeiten zur Reduzierung des Flächenverbrauches beschrieben und deren Umsetzbarkeit untersucht. Hierbei stehen der Flächenzertifikatehandel, der in einem Modellversuch seine Praxistauglichkeit bewiesen hat und die Etablierung des 30-Hektar-Ziels im ROG im Mittelpunkt.

\section{Theoretische Einführung}

Im 1. Abschnitt werden sowohl der Flächenverbrauch als auch die Problematik der Landdegradation im Kontext der Sustainable Development Goals betrachtet. Neben der Definition von Leitbegriffen wird der Bezug zum Konzept der planetarischen Leitplanken hergestellt. Sowohl die Sustainable Development Goals als auch das Konzept der planetarischen Leitplanken nehmen häufig Bezug auf den Boden als Ressource. Dieser ist allerdings nicht Schwerpunkt der Darlegungen, dennoch sind diese Aspekte vor dem Hintergrund folgender Überlegungen eingearbeitet. Ein geringerer Flächenverbrauch kann eine hinreichende Voraussetzung dafür sein, dass ein verbesserter Bodenschutz erreicht wird. Zwingend notwendig ist dies aber nicht. Beispielsweise kann eine Intensivierung der Landwirtschaft dazu führen, dass weniger Fläche in Anspruch genommen

Hendrik Hamacher, Mitarbeiter am Lehr- und Forschungsgebiet für Berg-, Umwelt- und Europarecht,

RWTH Aachen University,

Aachen, Deutschland wird und dennoch durch den Einsatz produktionssteigender Mittel wie Pestizide oder Düngemittel negative Folgen für den Boden entstehen. Eine mögliche Abgrenzung zwischen beiden Bereichen erläutert Seher. Untersuchungen zum Flächenverbrauch werden quantitativ gestaltet und qualitative Aspekte zum Bodenschutz i.d. R. weniger berücksichtigt. Sowohl Fokus der Raumplanung als auch der nachfolgenden Ausführungen ist auf die zweidimensionale Fläche gerichtet, also den Boden als Standort. Die unterschiedlichen Funktionen und Schutzaspekte des dreidimensionalen Systems Boden werden weniger betrachtet.

\subsection{Landdegradation und Flächenverbrauch im Kontext} der Sustainable Development Goals

Die Sustainable Development Goals sind eines der Hauptergebnisse der Konferenz für nachhaltige Entwicklung der Vereinten Nationen im Jahr 2012. ${ }^{2}$ Nach der Verabschiedung in der UN-Generalversammlung im September 2015, sind diese mit einer Laufzeit von 15 Jahren am 1. Januar 2016 in Kraft getreten. Erstmalig wurden 17, von allen Mitgliedstaaten akzeptierte Ziele etabliert, die wiederum in 169 Unterziele gegliedert sind. ${ }^{3}$ Mithilfe der Sustainable Development Goals soll Nachhaltigkeit in den Bereichen Ökonomie, Soziales und Ökologie vorangetrieben werden. Das Ziel 15 - Leben an Land - besagt beispielsweise, dass Wälder nachhaltiger bewirtschaftet oder die Bodendegradation beendet werden sollen. ${ }^{4}$ Mit dem Unterziel 15.3, das

1) Seher, Local Land and Soil News, 2014, 29, 31

2) Ehlers/Ginzky, Local Land and Soil News, 2014, 10, 11.

3) Vereinte Nationen: Transformation unserer Welt: die Agenda 2030 für nachhaltige Entwicklung, Stand 28.11.2019, abrufbar unter https://www.un.org/Depts/german/gv-70/band1/ar70001.pdf, S. 6f.

4) Ehlers/Ginzky, Local Land and Soil News, 2014, 10, 11. 
Bezug zum Flächen- und Bodenschutz nimmt, wird das Ganze weiter konkretisiert: ${ }^{5}$

By 2030, combat desertification, restore degraded land and soil, including land affected by desertification, drought and floods, and strive to achieve a land degradation-neutral world

Nach Wunder ist das Erreichen dieses Zieles nicht nur hinreichend für einen verbesserten Bodenschutz, sondern auch notwendig für das Erreichen anderer Sustainable Development Goals z.B. für die Ziele Maßnahmen zum Klimaschutz (Ziel 13) oder Nachhaltige Städte und Gemeinden (Ziel 11). ${ }^{6}$ Ehlers und Ginzky bezeichnen Böden aufgrund der Verflechtungen und Einflussbereiche auf andere Sustainable Development Goal als die entscheidende Voraussetzung für nachhaltige Entwicklung. ${ }^{7}$ Auch Deutschland hat sich im Rahmen der Nachhaltigkeitsstrategie zu einer Umsetzung der Ziele 15 und 15.3 verpflichtet. Unter anderem in Form des 30-Hektar-Ziels, das in Kapitel 2.5 betrachtet wird. ${ }^{8}$ Wie bereits die Definition zum Unterziel 15.3 zeigt, sind Land Degradation und Land Degradation Neutrality zentrale Begriffe. Das führt zu der Frage, wie genau werden diese Begriffe definiert? Damit haben sich bereits Wunder et al. in einer ausführlichen Studie zur Implementierung des Unterzieles 15.3 in Deutschland auseinandergesetzt. Dabei sind diese zu der Schlussfolgerung gekommen, dass im Deutschen keine etablierte Übersetzung existiert und die Begriffe gleichbedeutend verwendet werden. ${ }^{9}$ Beispielsweise kann der Begriff Land Degradation sowohl für Bodendegradation als auch für den Flächenverbrauch verwendet werden. ${ }^{10}$ Diesen Aspekt zeigt auch die deutsche Übersetzung von Wunder et al.:11

Verringerung oder Verlust der biologischen oder wirtschaftlichen Produktivität und der Vielseitigkeit von natürlich oder künstlich bewässerten Anbauflächen oder von Wiesen und Weideland, forstwirtschaftlich genutzten Flächen und Wäldern [...] infolge der Nutzung des Landes oder infolge eines einzelnen oder mehrerer miteinander verknüpfter Prozesse einschließlich solcher, die sich aus menschlichen Tätigkeiten und Siedlungsmustern ergeben

Als Vorteil dieser Definition sehen Wunder et al. die mehrdimensionale Ausrichtung an. ${ }^{12}$ Der Begriff der Land Degradation lenkt auch das Augenmerk auf das Konzept der Land Degradation Neutrality. Ziel ist es bis 2030 die weitere Degradation von Landflächen zu verhindern, aber gleichzeitig wirtschaftliche Entwicklungen zuzulassen. ${ }^{13}$ Unter anderem führen zunehmende Urbanisierungstendenzen zu einem steigenden Flächenverbrauch. In der Folge müssen an anderen Stellen Kompensationsmaßnahmen durchgeführt werden, die den Ausgleich von Ökosystemfunktionen oder Ökosystemdienstleistungen zum Ziel haben. ${ }^{14}$ In der Summe soll sich der Netto-Effekt auf null belaufen. ${ }^{15}$ Auch diese Definition ist umfassend und bezieht sowohl den Boden als auch die Fläche mit ein: ${ }^{16}$

Landdegradationsneutralität ist der Zustand, in dem die Menge und Qualität von für die Unterstützung von Ökosystemfunktionen und -dienstleistungen [...] innerhalb bestimmter zeitlicher und räumlicher Einheiten [...] stabil bleiben oder zunehmen

Kritik an den Sustainable Development Goals übt der Wissenschaftliche Beirat der Bundesregierung für Globale Umweltveränderungen (WBGU), weil das Konzept der planetarischen Leitplanken nicht beachtet wird und die Festlegung der Sustainable Development Goals ohne Berücksichtigung nicht zweckmäßig erscheint. Stattdessen sollten die Sustainable Development Goals mit Vorschlägen ergänzt werden, die den Ansatz der Leitplanken konkretisieren und detaillierter darlegen. ${ }^{17}$ Doch was verbirgt sich hinter dem Ansatz der planetarischen Leitplanken?

\subsection{Etablierung der Ressource Boden im Konzept} der planetarischen Leitplanken

Das Konzept der planetarischen Leitplanken wird seit 1994 vom WBGU entwickelt. Diese Grenzen werden auch als Leitplanken bezeichnet und eröffnen den Raum, in dem nachhaltige Entwicklung stattfinden kann. ${ }^{18} \mathrm{Um}$ das Leben zukünftiger Generationen zu ermöglichen, soll in den Leitplanken verblieben werden, weil das Überschreiten nicht nur regional begrenzt, sondern global Veränderungen hervorrufen kann. ${ }^{19} \mathrm{Um}$ diese globalen Veränderungen möglichst einzuschränken und zu kontrollieren, empfiehlt der WBGU ein Sustainable Development Goals mit dem Titel „Sicherung der Erdsysteme“. In diesem benennt der WBGU sechs globale Umweltveränderungen und dazugehörige Ziele. ${ }^{20}$ Häufig handelt es sich dabei um langsam ablaufende Veränderungen, wie zum Beispiel die Ozeanversauerung, das Einhalten des 2-Grad-Zieles oder auch das Stoppen der anhaltenden Land- und Bodendegradation. ${ }^{21}$ Im Hinblick darauf spricht sich auch der WBGU für das bereits in Kapitel 1.1 erläuterte Ziel der Land Degradation Neutrality aus. ${ }^{22}$ Diese Umweltschäden sind nur mit einem globalen Problemlösungsansatz und der Einbeziehung aller Verursacher möglich. ${ }^{23} \mathrm{Um}$ die Leitplanken einzuhalten, empfiehlt der WBGU die Etablierung von Steuerungselementen. ${ }^{24}$ In Bezug auf den Flächenverbrauch in Deutschland werden diese in Kapitel 3 näher betrachtet.

\section{Flächenverbrauch in Deutschland}

Als Flächenverbrauch definiert der Sachverständigenrat für Umweltfragen den täglichen Zuwachs der Siedlungs- und Verkehrsfläche. ${ }^{25} \mathrm{Zu}$ diesen Flächen zählen beispielsweise Wohnbau-, Industrie- und Gewerbeflächen oder Straßenund Bahnverkehr. Zwar werden Flächen für Bergbaube-

5) Vereinte Nationen: Work of the Statistical Commission pertaining to the 2030 Agenda for Sustainable Development, Stand 28.11.2019, abrufbar unter https://undocs.org/A/RES/71/313, S. 20 .

6) Wunder, Bodenschutz 2018, 58, 58.

7) Ehlers/Ginzky, Local Land and Soil News, 2014, 10, 10.

8) Wunder, Bodenschutz 2018, 58, 58

9) Wunder/Kaphengst/Frelih-Larsen/McFarland/Albrecht, Land Degradation Neutrality. Handlungsempfehlungen zur Implementierung des SDG-Ziels 15.3 und Entwicklung eines bodenbezogenen Indikators, 2018, S. 25

10) Wunder, Bodenschutz 2018, 58, 60

11) Wunder/Kaphengst/Frelih-Larsen/McFarland/Albrecht, (Fn. 9), S. 27.

12) Wunder/Kaphengst/Frelih-Larsen/McFarland/Albrecht, (Fn. 9), S. 25

13) Wunder, Bodenschutz 2018, S. 59.

14) Wunder/Kaphengst/Frelih-Larsen/McFarland/Albrecht, (Fn. 9), S. 28

15) Wunder, Bodenschutz 2018, S. 59.

16) Wunder/Kaphengst/Frelih-Larsen/McFarland/Albrecht, (Fn. 9), S. 28.

17) Wissenschaftlicher Beirat der Bundesregierung für globale Umweltveränderungen, Zivilisatorischer Fortschritt innerhalb planetarischer Leitplanken. Ein Beitrag zur SGD Debatte, 2014, S. 6.

18) Wissenschaftlicher Beirat der Bundesregierung für globale Umweltveränderungen, Welt im Wandel. Gesellschaftsvertrag für eine große Transformation. Hauptgutachten, 2011, S. 34.

19) Wissenschaftlicher Beirat der Bundesregierung für globale Umweltveränderungen, (Fn. 17), S. 6.

20) Wissenschaftlicher Beirat der Bundesregierung für globale Umweltveränderungen, (Fn. 17), S. 34.

21) Wissenschaftlicher Beirat der Bundesregierung für globale Umweltveränderungen, (Fn. 17), S. $6 \mathrm{f}$

22) Wissenschaftlicher Beirat der Bundesregierung für globale Umweltveränderungen, (Fn. 17), S. 6.

23) Wissenschaftlicher Beirat der Bundesregierung für globale Umweltveränderungen, (Fn. 17), S. 12.

24) Wissenschaftlicher Beirat der Bundesregierung für globale Umweltveränderungen, (Fn. 17), S. 36.

25) Sachverständigenrat für Umweltfragen, Umweltgutachten 2016 , 2016, S. 241. 
triebe, Tagebaue, Gruben und Steinbrüche in der statistischen Erfassung zu dieser Kategorie gezählt, aber nicht für den Zuwachs erfasst. Mitunter wird der Begriff Flächenverbrauch kritisiert, weil Fläche nicht verbraucht, sondern lediglich auf andere Weise genutzt werden kann. Stattdessen wird auf den Begriff Flächenneuinanspruchnahme verwiesen. Um Aufmerksamkeit für die Probleme und Folgen des Flächenverbrauches zu erregen, empfehlen Schröder et al. dennoch den Begriff Flächenverbrauch zu verwenden. Dieser Aspekt gilt insbesondere im Kontext öffentlicher und politischer Debatten. ${ }^{26}$ Dieser Empfehlung wird in den Ausführungen gefolgt. Die Nutzungsarten der Bodenfläche in Deutschland sind in die Kategorien Siedlung, Verkehr, Vegetation und Gewässer aufgeteilt. Mit einem Anteil von $84 \%$ hat die Kategorie Vegetation den größten Anteil an der Nutzungsarten. Innerhalb dessen entfallen 50,8\% auf die Landwirtschaft und 29,8\% auf Wälder. Die Wohnbauflächen haben in der Kategorie Siedlung einen Anteil von $42 \%$, gefolgt von Flächen für Gewerbe und Industrie mit $18 \%$. Auf Straßen und Wege entfallen in der Kategorie Verkehr $89 \%{ }^{27}$

\subsection{Flächenfunktionen}

Aufgrund vielfältiger Interessen treten in der Folge Flächennutzungskonkurrenzen auf. Diese Interessen beruhen auf den verschiedenen Funktionen, die der Fläche zugeordnet werden können. Dazu zählen die Naturraum-, Erholungs-, Produktions- und Trägerfunktion. Alle vier Funktionen können i.d.R. nicht auf derselben Fläche berücksichtigt werden, weil eine Nutzungsform zum ganzen oder teilweisen Ausschluss der anderen führt. Beispielsweise kann nicht der Lebensraum für Tiere und Pflanzen in seiner natürlichen Form erhalten werden (Naturraumfunktion), wenn die Fläche gleichzeitig als Grundlage für den Bau von Straßen oder Häusern (Trägerfunktion) genutzt wird. ${ }^{28}$

\subsection{Status quo des Flächenverbrauches in Deutschland}

In Anbetracht der Definition für den Flächenverbrauch rückt die Trägerfunktion mit seinen unterschiedlichen Nutzungstatbeständen in den Blickpunkt. Nach Angaben des Statistischen Bundesamtes hat jeder Einwohner 618 Quadratmeter Siedlungs- und Verkehrsfläche im Jahr 2016 in Anspruch genommen. ${ }^{29}$ Dieses Phänomen ist nicht erst seit Kurzem bekannt. Bereits 1974 hat der Sachverständigenrat für Umweltfragen in seinem ersten Gutachten den hohen Flächenverbrauch missbilligt und die negativen Auswirkungen dargestellt. ${ }^{30}$ Im Zeitraum von 1992 bis 2018 ist der Anteil der Siedlungs- und Verkehrsfläche von 40305 Quadratkilometer auf 49819 Quadratkilometer angestiegen. Dies entspricht einer Zunahme von 23,6\%. Siedlungsflächen verzeichnen mit $33 \%$ den größten Zuwachs. ${ }^{31}$

Auch der Sachverständigenrat für Umweltfragen kommt zu dem Schluss, dass Wohnen einer der maßgeblichen Einflussfaktoren für den Flächenverbrauch ist. Im Umweltgutachten aus dem Jahr 2016 wurden Daten aus dem Jahr 2013 einer differenzierten Betrachtung unterzogen. Für den Flächenverbrauch in den Kategorien Gebäude- und Freifläche sowie Betriebsfläche wurden 29 Hektar pro Tag ausgewiesen. ${ }^{32} \mathrm{Zu}$ den Freiflächen zählen diejenigen, die unbebaut und Zwecken der Gebäude untergeordnet sind. Beispiele sind Vor- und Hausgärten oder Stellplätze. ${ }^{33}$ Ferner wurden für die Kategorien Gewerbe- und Betriebsfläche jeweils 11 und 13 Hektar pro Tag angegeben. Demgegenüber stand ein Verlust von 23 Hektar pro Tag in der Kategorie Gebäude- und Freifläche Sonstige. In der Summe wurde ein Flächenverbrauch von 29 Hektar pro Tag festgestellt, ohne dass die Bedeutung der Gebäude- und Freiflächen erkennbar war. ${ }^{34}$ Bei der Dateninterpretation muss berücksichtigt werden, dass in den Vermessungsverwaltungen der Bundesländer seit einigen Jahren die Umstellung vom Automatisierten Liegenschaftsbuch (ALB) auf das Amtliche Liegenschaftskataster-Informationssystem (ALKIS) erfolgt. Infolgedessen wurde eine neue Nutzungsartensystematik eingeführt, deren Ergebnisse erstmals zum 31. Dezember 2016 vorgestellt wurden. ${ }^{35}$ Demzufolge ist eine 1:1 Überstellung zwischen den Nutzungsartenkatalogen nur bedingt möglich. ${ }^{36}$ Insbesondere wirkt sich die Umstellung auch auf die Berechnung der Siedlungs- und Verkehrsflächen aus. Im ALB Nutzungsartenkatalog setzt sich die Siedlungsfläche aus den Kategorien Gebäude- und Freifläche, Betriebsfläche (ohne Abbauland), Erholungsfläche und Friedhöfe zusammen. Im neuen Nutzungsartenkatalog wird der eigenständige Nutzungsartenbereich Siedlung angegeben. Allerdings kann dieser nicht deckungsgleich zur Berechnung der Siedlungsund Verkehrsfläche verwendet werden. Aufgrund dessen weist der Flächenverbrauch für 2016 eine Verzerrung auf. ${ }^{37}$ Für weitere Informationen wird an dieser Stelle auf den Methodenbericht zur Flächenerhebung verwiesen. ${ }^{38}$

Im Gegensatz dazu ist der Flächenverbrauch pro Tag seit 1992 stetig gesunken. Dieser wird als Mittelwert über vier Jahre angegeben, um konjunkturbedingte Schwankungen zu glätten. Betrug der Verbrauch zwischen 1997 und 2000 im Durchschnitt 129 Hektar pro Tag, ist dieser zwischen 2014 und 2017 auf 58 Hektar pro Tag zurückgegangen. ${ }^{39}$ Für die Jahre 2016 und 2017 kann lediglich der Mittelwert von 20132016 bzw. 2014-2017 verwendet werden. Hintergrund ist die Umstellung der Erhebungsmethode sowie die Einführung eines anderen Nutzungsartenkataloges. ${ }^{40}$ Doch ist fraglich, ob

26) Schröder/Huck/Haan, Transfer sozialer Innovationen. Eine zukunftsorientierte Fallstudie zur nachhaltigen Siedlungsentwicklung, 2011, S. 40.

27) Statistisches Bundesamt: Bodenfläche insgesamt nach Nutzungsarten in Deutschland, Stand 2.12.2019, abrufbar unter https:// www.destatis.de/DE/Themen/Branchen-Unternehmen/Landwirtschaft-Forstwirtschaft-Fischerei/Flaechennutzung/Tabellen/bodenflaeche-insgesamt.html.

28) Nachhaltigkeitsbeirat der Landesregierung Baden-Württemberg, Neue Wege zu einem nachhaltigen Flächenmanagement in BadenWürttemberg. Sondergutachten, 2004, S. 7.

29) Statistisches Bundesamt: Pressemitteilung Nr. 451 vom 15.11.2017, Stand 29.11.2019, abrufbar unter https://www.destatis.de/DE/ Presse/Pressemitteilungen/2017/11/PD17_409_412.html.

30) Sachverständigenrat für Umweltfragen, Umweltgutachten 2016, S. 241.

31) Umweltbundesamt: Siedlungs- und Verkehrsfläche, Stand 4.12.2019, abrufbar unter https://www.umweltbundesamt. de/daten/flaeche-boden-land-oekosysteme/flaeche/siedlungsverkehrsflaeche\#anhaltender-flachenverbrauch-fur-siedlungsund-verkehrszwecke-.

32) Sachverständigenrat für Umweltfragen, Umweltgutachten 2016, 2016, S. $246 f$.

33) Genesis-Online Datenbank: Erläuterung Gebäude und Freifläche, Stand 16.12.2019, abrufbar unter https://www-genesis.destatis.de/genesis/online/data?operation=ergebnistabelleInfo\&lev elindex $=3$ \&levelid $=1576528482707$.

34) Sachverständigenrat für Umweltfragen, Umweltgutachten 2016, 2016, S. $246 \mathrm{f}$.

35) Georg, Bayern in Zahlen. Fachzeitschrift für Statistik 2016, 771, 771

36) Georg, Bayern in Zahlen. Fachzeitschrift für Statistik 2016, 771, $773 f$.

37) Georg, Bayern in Zahlen. Fachzeitschrift für Statistik 2016, 771, $776 f$.

38) Statistische Ämter des Bundes und der Länder: Methodenbericht zur Flächenerhebung. Ausgabe 2018, Stand 16.12.2019, abrufbar unter https://www.statistikportal.de/sites/default/files/2019-06/ Methodenbericht_Fl\%C3\%A4chenerhebung.pdf.

39) Umweltbundesamt: Siedlungs- und Verkehrsfläche, Stand 4.12.2019, abrufbar unter https://www.umweltbundesamt. de/daten/flaeche-boden-land-oekosysteme/flaeche/siedlungsverkehrsflaeche\#anhaltender-flachenverbrauch-fur-siedlungsund-verkehrszwecke-.

40) Umweltbundesamt: Siedlungs- und Verkehrsfläche, Stand 4.12.2019, abrufbar unter https://www.umweltbundesamt. de/daten/flaeche-boden-land-oekosysteme/flaeche/siedlungsverkehrsflaeche\#anhaltender-flachenverbrauch-fur-siedlungsund-verkehrszwecke-. 
diese Daten einen Anlass zur Gelassenheit geben. Bereits jetzt wird deutlich, dass der Bevölkerungsrückgang mit einer Ausdehnung der Siedlungs- und Verkehrsfläche verbunden ist. Nach Prognosen für das Jahr 2050 werden sowohl ländliche als auch verstädterte Regionen in Deutschland am stärksten von Schrumpfungs- und Alterungsprozessen betroffen sein. Dahingegen ist Bevölkerungswachstum mit einer Abnahme des Flächenverbrauches verbunden, weil Flächen effektiver genutzt werden. ${ }^{41}$ Außerdem sind Baulandpreise in stagnierenden Regionen günstig, weil sich zugleich durch die Neuausweisung Einnahmen erhofft werden. Diese Annahmen zeigen sich auch dadurch, dass $70 \%$ des Flächenverbrauches außerhalb verdichteter Regionen stattfindet. Somit nimmt die Siedlungsfläche pro Kopf in ländlichen Regionen $\mathrm{zu}^{42}$

\subsection{Ursachen und Einflussfaktoren}

Der Flächenverbrauch unterliegt einem komplexen $\mathrm{Zu}-$ sammenspiel verschiedener Ursachen und Einflussfaktoren. ${ }^{43}$ Als Haupteinflussfaktor für den hohen Flächenverbrauch führen Schröder et al. den Neubau von Immobilien auf der grünen Wiese an. ${ }^{44}$

Damit werden Flächen bezeichnet, die noch nicht an die Infrastruktur angebunden sind und einen natürlichen Bodenzustand aufweisen. Diesen Flächen können unterschiedliche Nutzungen zugeführt werden, beispielswiese für Wohnen, Industrie oder Handel. ${ }^{45}$ Im Allgemeinen hat die Einwohnerzahl für das kommunale Einnahmesystem eine wichtige und nicht zu unterschätzende Bedeutung, da sie die Höhe des Anteils aus den Zuweisungen des Landeshaushaltes bestimmt. Auch die Ausweisung von neuen Gewerbeoder Industriegebieten ist in Folge der Gewerbesteuer eine wichtige Finanzierungsquelle für Kommunen. ${ }^{46}$ Darüber hinaus ist das Wachstum von Siedlungs- und Verkehrsflächen auch abhängig von konjunkturellen Bedingungen. ${ }^{47}$ In Folge einer verbesserten Wirtschaftslage nimmt der Flächenverbrauch aufgrund von Konsumgewohnheiten, Investitionen im Baugewerbe oder staatlichen Rahmenbedingungen und politischen Entscheidungen zu. ${ }^{48}$ Eine weitere Ursache für den Flächenverbrauch ist, dass Brachflächen durch höhere Kosten, planungsrechtliche Beschränkungen und Altlastenverdachtsfälle im Nachteil sind. Diese Aspekte fördern die Neuerschließung auf der grünen Wiese und betreffen insbesondere Gewerbeflächen. ${ }^{49}$

\subsection{Folgen}

Aus ökologischen Gesichtspunkten besteht die Notwendigkeit den Boden als Lebensraum für Tiere und Pflanzen zu erhalten. ${ }^{50}$ Insbesondere durch die Zunahme von Verkehrsflächen besteht die Gefahr von Zerschneidungseffekten für das Habitat von Tieren oder sogar die vollständige Isolierung von Populationen. ${ }^{51}$ Weiterhin ist problematisch, dass 43-50\% der Siedlungs- und Verkehrsflächen versiegelt sind. ${ }^{52}$ Die Versiegelung kann beispielsweise dazu führen, dass aufgrund der ausbleibenden Versickerung Grundwasservorräte nicht aufgefüllt werden oder das Überschwemmungsrisiko steigt. ${ }^{53}$ In Folge der ErschlieBung weiterer Flächen auf der grünen Wiese ist der Bau von Verkehrswegen oder die Errichtung von Versorgungsinfrastruktur nötig. Dieses Phänomen wird auch als sekundärer Flächenverbrauch bezeichnet. ${ }^{54}$ Mit der Erschließung neuer Flächen geht auch eine verkehrsinduzierende Wirkung einher. Diese zeichnet sich dadurch aus, dass ein erhöhtes Verkehrsaufkommen auftreten kann und Energieverbrauch oder Schadstoffemissionen ansteigen. Mitunter haben größere Wege auch soziale Auswirkungen, indem mobilitätsbezogener Stress auftritt oder Menschen älteren Alters nicht mehr teilhaben können ${ }^{55}$ Darüber hinaus kann die Neuausweisung auch zu Entmischungsprozessen führen, sodass in innerstädtischen Bereichen Leerstände auftreten oder die Bausubstanz verfällt. ${ }^{56}$
Auch aus ökonomischer Sicht ergeben sich - insbesondere im Zuge des demographischen Wandels - Nachhaltigkeitsprobleme. Für die Neuausweisung ist zunächst die Errichtung von Infrastruktur notwendig. Bei abnehmenden Bevölkerungszahlen könnte diese einerseits nicht mehr benötigt werden und andererseits durch das Ausbleiben von Grunderwerbssteuer nicht mehr finanzierbar sein. ${ }^{57}$ Folglich stellt die Neuausweisung eine riskante Vorleistung für Städte und Kommunen dar. ${ }^{58}$ Durch die Bewältigung größerer Strecken ergeben sich auch für Verbraucher, z. B. durch den Kauf eines Autos, ökonomische Risiken. ${ }^{59}$

\subsection{Das 30-Hektar-Ziel}

Im Jahr 2002 hat die Bundesregierung im Zusammenhang mit der Nachhaltigkeitsstrategie das Ziel formuliert, den Flächenverbrauch im Jahr 2020 auf 30 Hektar pro Tag zu senken. ${ }^{60}$ Seinen Ursprung hat dieses Ziel im Faktor 4 Konzept des Wuppertal-Instituts für Klima, Umwelt und Energie. Dieses besagt, dass der Ressourcenverbrauch um ein Viertel im Vergleich zum damaligen Verbrauch gesenkt werden sollte. Bei einem Flächenverbrauch von 120 Hektar für den gleitenden Vierjahressdurchschnitt von 1993-1996

41) Adrian/Bock/Bunzel/Preuß/Rakel, Instrumente zur Reduzierung der Flächeninanspruchnahme. Aktionsplan Flächensparen, 2018, 36.

42) Adrian/Bock/Bunzel/Preuß/Rakel, (Fn. 41), 37

43) Adrian/Bock/Bunzel/Preuß/Rakel, (Fn. 41), S. 40

44) Schröder/Huck/Haan, Transfer sozialer Innovationen. Eine zukunftsorientierte Fallstudie zur nachhaltigen Siedlungsentwicklung, 2011, S. 43.

45) Otparlik/Siemer/Ferber, Bezeichnungen und Definitionen für die Flächenerfassung, 2011, S. 4.

46) Schröder/Huck/Haan, (Fn. 44), S. 43; Adrian/Bock/Bunzel/Preuß/ Rakel, (Fn. 41), S. 40.

47) Umweltbundesamt, Hintergrundpapier: Flächenverbrauch, ein Umweltproblem mit wirtschaftlichen Folgen, 2004, S. 2.

48) Umweltbundesamt, (Fn. 47), S. 5

49) Umweltbundesamt, (Fn. 47), S. 2

50) Schröder/Huck/Haan, Transfer sozialer Innovationen. Eine zukunftsorientierte Fallstudie zur nachhaltigen Siedlungsentwicklung, 2011, S. 45

51) Umweltbundesamt, (Fn. 47), S. 2; Sachverständigenrat für Umweltfragen, Umweltgutachten 2016, 2016, S. 249.

52) Statistisches Bundesamt: Handelt es sich bei den Siedlungs- und Verkehrsflächen um versiegelte Flächen?, Stand 5.12.2019, abrufbar unter https://www.destatis.de/DE/Themen/BranchenUnternehmen/Landwirtschaft-Forstwirtschaft-Fischerei/FAQ/ siedlungsflaeche.html.

53) Umweltbundesamt: Bodenversiegelung, Stand 8.12.2019, abrufbar unter https://www.umweltbundesamt.de/daten/flaeche-bodenland-oekosysteme/boden/bodenversiegelung\#textpart-2https:// www.destatis.de/DE/Themen/Branchen-Unternehmen/Landwirtschaft-Forstwirtschaft-Fischerei/FAQ/siedlungsflaeche.html.

54) Schröder/Huck/Haan, Transfer sozialer Innovationen. Eine zukunftsorientierte Fallstudie zur nachhaltigen Siedlungsentwicklung, 2011, S. 43

55) Schröder/Huck/Haan, Transfer sozialer Innovationen. Eine zukunftsorientierte Fallstudie zur nachhaltigen Siedlungsentwicklung, 2011, S. 45

56) Basedow/Bolze/Gunreben/Jacob/Sbresny/Schrage/Steininger/Weichselbaum, Flächenverbrauch und Bodenversiegelung in Niedersachen, 2017, S. 10.

57) Schröder/Huck/Haan, Transfer sozialer Innovationen. Eine zukunftsorientierte Fallstudie zur nachhaltigen Siedlungsentwicklung, 2011, S. 45.

58) Sachverständigenrat für Umweltfragen, Umweltgutachten 2016 , 2016, S. 252.

59) Schröder/Huck/Haan, Transfer sozialer Innovationen. Eine zukunftsorientierte Fallstudie zur nachhaltigen Siedlungsentwicklung, 2011, S. 45

60) Bundesregierung: Perspektiven für Deutschland. Unsere Strategien für eine nachhaltige Entwicklung, Stand 12.12.2019, abrufbar unter https://www.nachhaltigkeit.info/media/1326188329 phpYJ8KrU.pdf. 
entspricht dies 30 Hektar. In Anbetracht der vorherigen Ausführungen stellt sich jedoch die Frage, ob dieses Ziel bis 2020 erreicht werden kann. Das Bundesinstitut für Bau-, Stadt- und Raumforschung (BBSR) prognostiziert einen Flächenverbrauch von 48-50 Hektar pro Tag im Jahr 2020, sodass das 30-Hektar-Ziel nicht erreicht wird. ${ }^{61}$ Auch in der Politik besteht keine Zuversicht am Erreichen des Zieles. So wurde in der Neuauflage der Deutschen Nachhaltigkeitsstrategie aus dem Jahr 2016 das Ziel weiterentwickelt und beabsichtigt, dass der Flächenverbrauch auf 30 Hektar minus X pro Tag bis 2030 sinken soll. ${ }^{62}$ Für das Jahr 2030 hingegen bestehen gute Aussichten. Das Umweltbundesamt prognostiziert bei gleichbleibender Abnahme, dass sogar ein 20-Hektar Ziel erreicht werden kann. ${ }^{63}$ Nichtsdestotrotz könnte bei Erreichen des Zieles bis in das Jahr 2030 eine Fläche verloren gehen, die größer ist als das Saarland. ${ }^{64}$ Daher gilt es zu betrachten, welche Instrumente und Möglichkeiten bestehen, den Flächenverbrauch zu verringern.

\section{Möglichkeiten und Steuerungselemente zur Verringerung des Flächenverbrauches in Deutschland}

Adrian et al. kommen in ihrem Gutachten zu dem Ergebnis, dass sowohl auf kommunaler als auch regionaler Ebene eine Vielzahl an Instrumenten zur Verfügung steht, um den Flächenverbrauch zu verringern. ${ }^{65}$ Allerdings schaffen diese keinen Anreiz zum Flächensparen, sodass auch die Etablierung neuer Instrumente nötig ist. Im Allgemeinen können diese nach informatorischen, strukturierenden, regulativen und ökonomischen Ansatzpunkten unterschieden werden. ${ }^{66} \mathrm{Im}$ Rahmen einer flächenpolitischen Strategie haben Adrian et al. drei Bausteine herausgearbeitet. Dazu zählen die wirksame Begrenzung und Steuerung des Flächenverbrauches, die Erhöhung der Effizienz der Flächennutzung und die Mobilisierung von Bauland und Gebäuden im Bestand. ${ }^{67}$

\subsection{Flächenkreislaufwirtschaft und Kontingentierung}

Die Gesamtstrategie ist auch Voraussetzung für das Erreichen der Flächenkreislaufwirtschaft. ${ }^{68}$ Dieser Ansatz ist im Klimaschutzplan 2050 der Bundesregierung festgeschrieben und das zentrale Element im Handlungsbereich Fläche. So soll der Flächenverbrauch bis 2050 auf Netto Null gesenkt werden. ${ }^{69}$ Im Rahmen dessen sollen zunächst Potenziale im Bestand, also Baulücken und Brachflächen wiedergenutzt werden. Gleichzeitig soll die Inanspruchnahme neuer Flächen unter bestimmten Voraussetzungen möglich sein. ${ }^{70} \mathrm{Um}$ den Flächenverbrauch wirksam zu begrenzen und steuern, ist die Kontingentierung ein zentrales Element. ${ }^{71}$ Über einen Aushandlungsprozess zwischen Bund und Ländern könnte zunächst eine Höchstmenge, z. B. in Form des 30-HektarZiels an Bauland festgelegt und dann über einen Verteilschlüssel auf die Bundesländer übertragen werden. ${ }^{72}$ Gleichzeitig ist die Kontingentierung elementare Voraussetzung für die Einführung eines Flächenzertifikatehandels. ${ }^{73}$

\subsection{Flächenzertifikatehandel}

Zwischen 2013 und 2017 wurde im Auftrag des Umweltbundesamtes ein Modellversuch zum Flächenzertifikatshandel durchgeführt. Mit 87 Kommunen aus Deutschland wurde der Handel zwischen 2014 und 2028 simuliert. Ziel war es, zu überprüfen, ob der Flächenverbrauch gesenkt und die Innenentwicklung gestärkt werden können. ${ }^{74}$ Insbesondere vor dem Hintergrund der Neuausweisung von Gebieten im Außenbereich vieler Gemeinden und der Kontingentierung erfährt der Flächenzertifikatehandel eine besondere Bedeutung. Der Modellversuch bestand aus einer kommunalen Fallstudie und einem kontrollierten Feldexperiment. Im Rahmen des Experimentes haben die Kom- munen alle Planungen für deren Baulandentwicklungen im Zeitraum von 2014-2028 eingebracht. Auf Grundlage dessen wurde der Handel mit den Zertifikaten in einer Langzeitsimulation erprobt. ${ }^{75}$ Dahingegen hatte die Fallstudie nur eine ergänzende Funktion für das Experiment. Beispielsweise wurde in Workshops untersucht, wie der Handel die Bauleitplanung konterkariert. ${ }^{76}$

Sobald eine Kommune Flächen im Außenbereich ausweist, besteht die Notwendigkeit Zertifikate zu besitzen. Ein Zertifikat entspricht 1000 Quadratmeter Bauland. Übersteigt der Flächenbedarf die Anzahl der vorhandenen Zertifikate, ist der Erwerb weiterer notwendig. Dahingegen können Kommunen nicht verwendete Zertifikate über eine Handelsplattform anderen Kommunen zur Verfügung stellen und, sofern Bedarf besteht, verkaufen. Die Anzahl der Zertifikate orientiert sich am vorgegebenen Kontingent wie z.B. dem 30-Hektar-Ziel. ${ }^{77}$

Weiterhin wurden im Modellversuch weiße Zertifikate eingeführt. Kommunen können zusätzliche Zertifikate erwerben, wenn bestehende Siedlungs- und Verkehrsflächen wieder für einen anderen Zweck - unter Maßgabe qualitativer Aspekte - zugeführt werden z. B. als Landwirtschaftsfläche. In Vorbereitung auf das Experiment stellte sich heraus, dass $83 \%$ der Flächen zertifikatspflichtig sind und sich somit

61) Bundesinstitut für Bau-, Stadt- und Raumforschung: Das 30-Hektar-Ziel der Nachhaltigkeitsstrategie, Stand 18.12.2019, abrufbar unter https://www.bbsr.bund.de/BBSR/DE/Raumentwicklung/Flaechenpolitik/Projekte/30HektarZiel/30_ha_ ziel.html?nn=413116.

62) Bundesregierung: Deutsche Nachhaltigkeitsstrategie. Neuauflage 2016, Stand 19.12.2019, abrufbar unter https://www. bundesregierung.de/resource/blob/975292/730844/3d30c6c2875a9a08d364620ab7916af6/deutsche-nachhaltigkeitsstrategie-neuauflage-2016-download-bpa-data.pdf?download=1.

63) Umweltbundesamt: Siedlungs- und Verkehrsfläche, Stand 4.12.2019, abrufbar unter https://www.umweltbundesamt. de/daten/flaeche-boden-land-oekosysteme/flaeche/siedlungsverkehrsflaeche\#anhaltender-flachenverbrauch-fur-siedlungsund-verkehrszwecke-.

64) Umweltbundesamt: Siedlungs- und Verkehrsfläche, Stand 4.12.2019, abrufbar unter https://www.umweltbundesamt. de/daten/flaeche-boden-land-oekosysteme/flaeche/siedlungsverkehrsflaeche\#anhaltender-flachenverbrauch-fur-siedlungsund-verkehrszwecke-.

65) Adrian/Bock/Bunzel/Preuß/Rakel, Instrumente zur Reduzierung der Flächeninanspruchnahme. Aktionsplan Flächensparen, 2018, S. 92.

66) Adrian/Bock/Bunzel/Preuß/Rakel, Instrumente zur Reduzierung der Flächeninanspruchnahme. Aktionsplan Flächensparen, 2018, S. 53.

67) Adrian/Bock/Preuß, Nachrichten Magazin der Akademie für Raumforschung und Landesplanung 2016, 26.

68) Adrian/Bock/Bunzel/Preuß/Rakel, Instrumente zur Reduzierung der Flächeninanspruchnahme. Aktionsplan Flächensparen, 2018, S. 92.

69) Bundesministerium für Umwelt, Naturschutz und nukleare Sicherheit, Klimaschutzplan 2050. Klimapolitische Grundsätze und Ziele der Bundesregierung, 2016, S. $67 \mathrm{f}$.

70) Adrian/Bock/Bunzel/Preuß/Rakel, Instrumente zur Reduzierung der Flächeninanspruchnahme. Aktionsplan Flächensparen, 2018, S. 92.

71) Adrian/Bock/Bunzel/Preuß/Rakel, Instrumente zur Reduzierung der Flächeninanspruchnahme. Aktionsplan Flächensparen, 2018, S. 100.

72) Adrian/Bock/Bunzel/Preuß/Rakel, Instrumente zur Reduzierung der Flächeninanspruchnahme. Aktionsplan Flächensparen, 2018, S. 98.

73) Adrian/Bock/Bunzel/Preuß/Rakel, Instrumente zur Reduzierung der Flächeninanspruchnahme. Aktionsplan Flächensparen, 2018, S. 100.

74) Grimski, Bodenschutz 2018, 44, 44.

75) Grimski, Bodenschutz 2018, 44, 46.

76) Grimski, Bodenschutz 2018, 44, 47.

77) Grimski, Bodenschutz 2018, 44, 45. 
im Außenbereich befinden. Wiederum 83\% dieser Flächen sind für Wohnbauflächen auf der grünen Wiese vorgesehen. Die verfügbaren und nicht zertifikatspflichtigen Flächen im Innenbereich entsprechen ungefähr den Flächen, die Kommunen für Bauvorhaben im Außenbereich eingeplant haben. ${ }^{78}$ Das Flächennutzungspotenzial beläuft sich nach Berechnungen von Goetzke et al. aber lediglich auf $70 \% .{ }^{79}$ Für den Fall, dass diese Flächen für den Wohnbau genutzt werden, könnten $85 \%$ der bis 2028 geplanten Wohnbauvorhaben auf Innenentwicklungsflächen vollzogen werden. ${ }^{80} \mathrm{Da}-$ mit Kommunen ökonomische Aspekte umfangreicher bei der Neuausweisung in den Blick nehmen, wurden zusätzlich Fiskalwerte erhoben. ${ }^{81}$ Für weitere Informationen wird an dieser Stelle auf die Publikation Modellversuch Flächenzertifikatehandel vom Umweltbundesamt verwiesen, die im Oktober 2019 erschienen ist.

Im Ergebnis ist der Flächenverbrauch im Außenbereich um mehr als die Hälfte gesunken. Lediglich $52 \%$ der Flächen wurden für Vorhaben in Anspruch genommen. Dahingegen verdeutlichen die Ergebnisse die grassierende Bedeutung der Innenentwicklung. Im Rahmen der Experimente wurden $96 \%$ der Flächen erhalten. ${ }^{82}$ Die Effizienz des Zertifikatmarktes wurde von allen Teilnehmern mit Werten zwischen $86 \%$ und $92 \%$ bewertet, weil der Handel durch Einnahmen einen positiven ökonomischen Nutzen impliziert. ${ }^{83}$ Dieser Aspekt wird auch in den Ergebnissen der fiskalischen Wirkungsanalyse deutlich. Einen negativen Fiskalwert haben $35 \%$ der Flächen aufgewiesen, die Einnahmen waren also geringer als die Ausgaben. Wiederum $92 \%$ dieser Flächen lagen im Außenbereich. Einerseits sind infolge geringerer Bebauungsdichten die Preise niedrig und andererseits die zusätzlichen Erschließungskosten für Infrastruktur hoch. ${ }^{84}$

Doch besteht überhaupt eine Rechtsgrundlage und wenn ja, welche? Und wird ferner nicht die kommunale Planungshoheit, die nach Art. 28 Abs. 2 GG garantiert ist, verletzt? Dieser Frage widmen sich Köck et al. in einem Sachverständigengutachten im Auftrag des Umweltbundesamtes, das zum gegenwärtigen Zeitpunkt noch nicht erschienen ist. ${ }^{85}$ Die Zwischenergebnisse sind in einem Artikel in der Zeitschrift für Umweltrecht veröffentlicht. Köck et al. kommen zu dem Ergebnis, dass der Flächenzertifikatehandel der Selbstverwaltungsgarantie nicht zuwiderläuft und der unantastbare Kernbereich nicht tangiert wird. Aufgrund der Gemeinwohlgründe bestehen für den Eingriff außerhalb des Kernbereiches verschiedene Rechtfertigungsgründe. ${ }^{86}$ Da der Handel an die Kommunen adressiert ist, ist eine Integration in das Baugesetzbuch (BauGB) möglich. Dagegen spricht die Handelskomponente, die nicht notwendigerweise für die Integration in das BauGB sprechen. Infolgedessen raten Köck et al. an - unter Beachtung rechtstechnischer Anpassungen im BauGB - ein einheitliches Flächenzertifikatehandelsgesetz zu etablieren. ${ }^{87}$

\subsection{Stärkung der Innenentwicklung}

Die Ergebnisse des Flächenzertifikathandels geben Anlass die Möglichkeiten zur Stärkung der Innenentwicklung näher zu betrachten. Die Mobilisierung von Flächen im Bestand ist eine wichtige Stellschraube für die Innenentwicklung. ${ }^{88}$ Nach Berechnungen des BBSR beträgt das Innenentwicklungspotenzial zwischen 120000 und 166000 Hektar. ${ }^{89} \mathrm{Um}$ das Innenentwicklungspotenzial zu verbessern und den größtmöglichen Teil auszuschöpfen, gibt es verschiedene Ansätze. Eine Möglichkeit ist die Grunderwerbssteuer in eine Neubesiedlungssteuer zu überführen. ${ }^{90}$ Die Grunderwerbssteuer wird beim Erwerb von Eigentum sowohl für das Grundstück als auch das Gebäude fällig und von den Bundesländern erhoben. Dies gilt auch für Bestandsimmobilien sowie den Zwischenerwerb. Aufgrund der Tatsache, dass Immobilienpreise und die anteilige Grunderwerbssteuer im Umland geringer sind, wird die Wiedernutzbar- machung gegenüber dem Neubau auf der grünen Wiese eindeutig benachteiligt. Durch eine Neubesiedlungssteuer würden Bestandsobjekte und der kommunale Zwischenerwerb von Immobilien befreit und die Neuerschließung mit einem höheren Satz besteuert. Nebenbei könnte dies auch zu Anreizen für eine effektivere Ausnutzung des Grundstücks führen. Für die praktische Umsetzung kommen verschiedene Modelle infrage, deren Beitrag zur Reduzierung jeweils variiert. Fakt ist, dass alle Modelle zu einer Reduzierung führen. ${ }^{91}$ Problematisch ist, dass die Grunderwerbssteuer für die Bundesländer eine große Einnahmequelle darstellt. Im Jahr 2016 betrugen diese 12,4 Milliarden Euro. Nichtsdestotrotz sei dieses Instrument laut Adrian et al. sehr wichtig für das Flächensparen. ${ }^{92}$ Als ergänzender Ansatz für eine Reform der Grundsteuer wird die Einführung eines zonierten Satzungsrechtes diskutiert. In Folge fehlender Bereitschaft der Eigentümer, ihre Grundstücke zu entwickeln, wird die Entwicklung dieser Flächen oftmals gehemmt. ${ }^{93}$ Ein Grund liegt in der größeren Nachfrage nach Fläche, die eine Preissteigerung von städtischen Böden und Immobilienpreisen zur Folge hat. Hierdurch kommt es zu Spekulationen, um hohe Gewinne zu erzielen. ${ }^{94}$ Die Flächen im Innenbereich unterliegen der Grundsteuer B und sind demnach steuerlich kaum belastet. Eine Anhebung mit einem gesonderten Satz könnte die Steuerbelastung der Eigentümer erhöhen und somit die schnellere Entwicklung der Flächen begünstigen. Bei einer hohen Belastung wird die Mobilisierung von Bestandsflächen erwartet. Dennoch müssen sowohl Praxistauglichkeit als auch rechtliche Zulässigkeit untersucht werden. ${ }^{95}$

Ferner kann die Ökologisierung des kommunalen Finanzausgleiches ein Anreiz sein, den Flächenverbrauch zugunsten einer stärkeren Innenentwicklung zu orientieren. ${ }^{96}$ In der gegenwärtigen Konzeption werden großzü-

78) Grimski, Bodenschutz 2018, 44, 48.

79) Goetzke/Schlump/Hoymann/Beckmann/Dosch, Flächenverbrauch, Flächenpotentiale und Trends 2030, 2014, S. 10.

80) Grimski, Bodenschutz 2018, 44, 48.

81) Grimski, Bodenschutz 2018, 44, 48f.

82) Grimski, Bodenschutz 2018, 44, 49.

83) Grimski, Bodenschutz 2018, 44, 50.

84) Grimski, Bodenschutz 2018, 44, $48 \mathrm{f}$.

85) Grimski, Bodenschutz 2018, 44, $51 \mathrm{f}$

86) Köck/Bovet/Tietz, Zeitschrift für Umweltrecht 2018, 67, 72

87) Köck/Bovet/Tietz, Zeitschrift für Umweltrecht 2018, 67, 72.

88) Adrian/Bock/Bunzel/Preuß/Rakel, Instrumente zur Reduzierung der Flächeninanspruchnahme. Aktionsplan Flächensparen, 2018, S. 105.

89) Bundesinstitut für Bau-, Stadt- und Raumforschung, Innenentwicklungspotenziale in Deutschland - Ergebnisse einer bundesweiten Umfrage und Möglichkeiten einer automatisierten Abschätzung, 2013, S. 68.

90) Adrian/Bock/Bunzel/Preuß/Rakel, Instrumente zur Reduzierung der Flächeninanspruchnahme. Aktionsplan Flächensparen, 2018, S. 110.

91) Adrian/Bock/Bunzel/Preuß/Rakel, Instrumente zur Reduzierung der Flächeninanspruchnahme. Aktionsplan Flächensparen, 2018, S. 110.

92) Adrian/Bock/Bunzel/Preuß/Rakel, Instrumente zur Reduzierung der Flächeninanspruchnahme. Aktionsplan Flächensparen, 2018, S. 111.

93) Adrian/Bock/Bunzel/Preuß/Rakel, Instrumente zur Reduzierung der Flächeninanspruchnahme. Aktionsplan Flächensparen, 2018, S. 106.

94) Wissenschaftlicher Beirat der Bundesregierung für globale Umweltveränderungen, Der Umzug der Menschheit. Die transformative Kraft der Städte. Hauptgutachten, 2016, S. 174.

95) Adrian/Bock/Bunzel/Preu//Rakel, Instrumente zur Reduzierung der Flächeninanspruchnahme. Aktionsplan Flächensparen, 2018, S. 106.

96) Adrian/Bock/Bunzel/Preuß/Rakel, Instrumente zur Reduzierung der Flächeninanspruchnahme. Aktionsplan Flächensparen, 2018, S. 114 . 
gig Wohngebiete ausgewiesen, um die Einwohnerzahl und damit verbundene Finanzzuweisungen zu erhöhen. Eine Vorhaltung von Flächen für ökologische Funktionen oder sogar die Aufwertung von Siedlungsflächen wird nicht beachtet. ${ }^{97}$ Umstritten sind aber zum einen verfahrenspraktische Angelegenheiten und verfassungsrechtliche Hürden. ${ }^{98}$

\subsection{Rechtliche Verankerung des 30-Hektar-Ziels}

Die Raumordnung verhindert als einziges Rechtsinstrument die planlose Zersiedlung des Bundesgebietes. ${ }^{99}$ Aufgrund der Wirkung im gesamten Bundesgebiet - jeweils unter Berücksichtigung regionaler und lokaler Besonderheiten - spricht sich Gröhn für eine Verankerung des 30-Hektar-Ziels im ROG aus. ${ }^{100}$ Bereits zum gegenwärtigen Zeitpunkt wird in $\$ 2$ Abs. 2 Nr. 6 ROG die Vorgabe gemacht, die Flächeninanspruchnahme im Freiraum zu begrenzen. In Folge der Verankerung würde eine weitere Konkretisierung erreicht und umweltbezogene Vorsorgegrundsätze gestärkt werden. Andererseits muss berücksichtigt werden, wie das Ziel auf nachfolgende Planungsebenen übertragen werden kann. ${ }^{101}$ Sobald das 30-Hektar-Ziel gesetzlich festgeschrieben wird, würde dieses in landesweite und regionale Raumordnungs- sowie kommunale Bauleitpläne einfließen und einen verbindlichen Rahmen vorgeben. ${ }^{102}$ Problematisch ist, dass die Vorgabe für konkrete, parzellenscharfe Flächennutzungen dem Gesetzgeber auf Bundesebene untersagt ist. ${ }^{103}$ Zwar würde die Quotenregelung nicht unzulässig in die Rechtsstellung der Länder eingreifen, aber die Verankerung könnte $\mathrm{zu}$ Widerständen in den Kommunen führen und durch die Abweichungsgesetzgebung (Art. 72 Abs. 3 GG) konterkariert werden. ${ }^{104} \mathrm{Um}$ mehr Akzeptanz zu schaffen, ist es sinnvoll die Bundesländer mit einzubeziehen. ${ }^{105} \mathrm{Im}$ Allgemeinen sollte die Flächenpolitik kein singuläres Politikfeld darstellen, sondern auf allen Ebenen der räumlichen Planung Beachtung finden. ${ }^{106}$

\section{Fazit}

Einer der Meilensteine für nachhaltige Entwicklung sind die Sustainable Development Goals, die mit einer Laufzeit von 15 Jahren am 1. Januar 2016 wirksam geworden sind. In dieser Form haben sich zum ersten Mal alle Mitgliedstaaten der UN auf 17 Ziele und 169 Unterziele geeinigt, um nachhaltige Entwicklung weltweit $\mathrm{zu}$ intensivieren. Bezug zum Flächen- und Bodenschutz nimmt das Unterziel 15.3, das die Begriffe der Land Degradation und Land Degradation Neutrality hervorhebt.

Eine exakte Übersetzung für den Begriff Land Degradation ins Deutsche besteht nicht, so kann dieser sowohl für Bodendegradation als auch den Flächenverbrauch verwendet werden. Auch Deutschland hat sich im Rahmen der Nachhaltigkeitsstrategie zu einer ambitionierten Umsetzung der Sustainable Development Goals verpflichtet. Dazu zählt auch das Erreichen des 30-Hektar-Ziels, das besagt, den täglichen Flächenverbrauch bis 2030 auf unter 30 ha pro Tag zu senken. Als Flächenverbrauch wird der tägliche Zuwachs der Siedlungs- und Verkehrsfläche definiert. Zwar ist der tägliche Flächenverbrauch zwischen 1993 und 2017 von 120 auf 58 ha pro Tag rückläufig, dahingegen ist der absolute Anteil der Siedlungs- und Verkehrsfläche zwischen 1992 und $2018 \mathrm{um}$ mehr als $9000 \mathrm{~km}^{2}$ angestiegen. Wenngleich das 30-Hektar-Ziel bis 2030 vermutlich erreicht wird, würde eine Fläche verbraucht werden, die so groß ist wie das Saarland. Folglich wäre es wichtig, eine Flächenkreislaufwirtschaft zu erreichen, sodass nur noch bedingt neue Flächen verbraucht werden. Zu dieser hat sich die Deutsche Bundesregierung im Klimaschutzplan für das Jahr 2050 verpflichtet.

Die Gründe für den anhaltenden Flächenverbrauch liegen insbesondere im kommunalen Finanzierungssystem.
Beispielsweise weisen Gemeinden neue Flächen für Wohnen aus, weil die Einwohnerzahl die Zuweisungen aus dem Landeshaushalt bestimmt, oder diese sich über die Ausweisung neuer Industriegebiete Einnahmen aus der Gewerbesteuer erhoffen. Aufgrund der Multifunktionalität der Fläche treten diverse Folgen auf. Neben der Zerschneidung von Lebensräumen für Tiere und Pflanzen führt die Neuausweisung einerseits zu einem höheren Verkehrsaufkommen. Andererseits nehmen Kommunen ökonomische Risiken in Kauf, weil angesichts von Schrumpfungs- und Abwanderungsprozessen die Refinanzierbarkeit der getätigten Investitionen in die Infrastruktur nicht gegeben ist. Bis 2050 sind in Deutschland von Abwanderungsprozessen vorwiegend ländliche und verstädterte Regionen in Ostdeutschland betroffen. Neben der Stärkung der Innenentwicklung, erweisen sich die rechtliche Verankerung des 30-Hektar-Ziels und die Einführung eines Flächenzertifikatehandels als vielversprechend. Mit dem 30-Hektar-Ziel würde zunächst über einen Verteilschlüssel ein festes Kontingent vorgegeben werden. Dies wiederum ist elementar für die Einführung des Flächenzertifikatehandels. Die Praxistauglichkeit dessen wurde im Modellversuch zwischen 2013 und 2017 unter Beweis gestellt. Lediglich an 52\% der Flächen im Außenbereich wurden festgehalten, wohingegen $96 \%$ der Flächen im Innenbereich erhalten wurden. Wichtig für die erfolgsversprechende Innenentwicklung wären ökonomische Anreize wie die Einführung einer Neubesiedlungssteuer oder eines zonierten Satzungsrechtes. Gegenwärtig wäre es wichtig die rechtlichen Konstellationen anzupassen und sowohl ein Flächenzertifikatehandelsgesetz zu erlassen als auch das 30-Hektar-Ziel im ROG $\mathrm{zu}$ verankern.

Open Access. Dieser Artikel wird unter der Creative Commons Namensnennung 4.0 International Lizenz veröffentlicht, welche die Nutzung, Vervielfältigung, Bearbeitung, Verbreitung und Wiedergabe in jeglichem Medium und Format erlaubt, sofern Sie den/die ursprünglichen Autor(en) und die Quelle ordnungsgemäß nennen, einen Link zur Creative Commons Lizenz beifügen und angeben, ob Änderungen vorgenommen wurden.

Die in diesem Artikel enthaltenen Bilder und sonstiges Drittmaterial unterliegen ebenfalls der genannten Creative Commons Lizenz, sofern sich aus der Abbildungslegende nichts anderes ergibt. Sofern das betreffende Material nicht unter der genannten Creative Commons Lizenz steht und die betreffende Handlung nicht nach gesetzlichen Vorschriften erlaubt ist, ist für die oben aufgeführten Weiterverwendungen des Materials die Einwilligung des jeweiligen Rechteinhabers einzuholen.

Weitere Details zur Lizenz entnehmen Sie bitte der Lizenzinformation auf http://creativecommons.org/licenses/by/4.0/deed.de.

Open access funding provided by Projekt DEAL.

97) Adrian/Bock/Bunzel/Preuß/Rakel, Instrumente zur Reduzierung der Flächeninanspruchnahme. Aktionsplan Flächensparen, 2018, S. 113.

98) Adrian/Bock/Bunzel/Preuß/Rakel, Instrumente zur Reduzierung der Flächeninanspruchnahme. Aktionsplan Flächensparen, 2018, S. 114.

99) Gröhn, NuR 2016, 78, 81.

100) Gröhn, NuR 2016, 78, 80.

101) Gröhn, NuR 2016, 78, 81.

102) Adrian/Bock/Bunzel/Preuß/Rakel, Instrumente zur Reduzierung der Flächeninanspruchnahme. Aktionsplan Flächensparen, 2018, S. 100

103) Gawron 2007, zitiert nach Gröhn, NuR 2016, 78, 81.

104) Gröhn, NuR 2016, 78, 82; Adrian/Bock/Bunzel/Preuß/Rakel, Instrumente zur Reduzierung der Flächeninanspruchnahme. Aktionsplan Flächensparen, 2018, S. 100.

105) Gröhn, NuR 2016, 78, 82.

106) Adrian/Bock/Bunzel/Preuß/Rakel, Instrumente zur Reduzierung der Flächeninanspruchnahme. Aktionsplan Flächensparen, 2018, S. 96. 